\title{
Cost-effectiveness and budget impact of dolutegravir/lamivudine for treatment of human immunodeficiency virus (HIV-1) infection in the United States
}

Karin Butler, MSc; Sarah-Jane Anderson, PhD; Olivia Hayward, PhD; Ian Jacob, MSc; Yogesh Suresh Punekar, PhD; Lee Alexandra Evitt, MSc; and Alan Oglesby, MPH

\section{What is already known about this subject}

- Combination antiretroviral therapy (ART) has helped people living with HIV (PLHIV) achieve and sustain longterm viral suppression turning HIV into a manageable chronic condition.

- As PLWHIV live longer, the costs of HIV treatment and management are increasing.

- Simplified 2-drug ART regimens that provide equivalent efficacy while reducing ART exposure to standard of care 3-drug regimens are now endorsed by HIV clinical guidelines and have the potential for reduced costs.

\section{ABSTRACT}

BACKGROUND: Dolutegravir(DTG)/ lamivudine(3TC) is the first 2-drug regimen recommended as an initial treatment for people living with HIV (PLHIV).

OBJECTIVE: To assess the cost-effectiveness and potential budget impact of DTG/3TC in the US healthcare setting.

METHODS: A previously published hybrid decision-tree and Markov cohort state transition model was adapted to estimate the incremental costs and health outcome benefits over a patients' lifetime. DTG/3TC was compared with current standard of care in

\author{
What this study adds \\ - This analysis estimates the cost- \\ effectiveness and potential economic \\ impact of dolutegravir(DTG)/ \\ lamivudine(3TC), the first 2-drug \\ regimen recommended as an initial \\ treatment or stable-switch option for \\ people living with HIV (PLHIV) in the \\ United States. \\ - Results suggest that DTG/3TC, with \\ its comparable efficacy, durability, \\ and lower drug acquisition costs, is \\ predicted to offer significant cost \\ savings compared to other common \\ standard of care HIV treatment \\ regimens for both treatment naive \\ PLWH and as a treatment switching \\ option for virologically suppressed \\ patients on existing treatment.
}

treatment naive and treatment experienced virologically suppressed PLHIV. Health states included in the model were based upon virologic response and CD4 cell count, with death as an absorbing state. Clinical data was informed by the Phase III GEMINI 1 and 2 clinical trials, a published network metaanalysis (NMA) in treatment-naive patients and the Phase III TANGO clinical trial in treatment experienced patients. Costs and utilities were informed by published data and discounted annually at a rate of $3 \%$. A separate 5 -year budget impact analysis was conducted assuming 5\%-15\% uptake in eligible treatment naive and $10 \%-30 \%$ uptake in eligible treatment experienced patients.

\section{Author affiliations}

Karin Butler, MSc; Olivia Hayward, PhD; Ian Jacob, MSc; Health Economics \& Outcomes Research Ltd, Cardiff, United Kingdom. Sarah-Jane Anderson, PhD; GlaxoSmithKline, Brentford, United Kingdom. Yogesh Suresh Punekar, PhD; Lee Alexandra Evitt, MSc; ViiV Healthcare, Brentford, United Kingdom; Alan Oglesby, MPH; ViiV Healthcare, NC.

AUTHOR CORRESPONDENCE:

Alan Oglesby, 919.561.2766;

alan.k.oglesby@viivhealthcare.com

J Manag Care Spec Pharm. 2021;27(7):891-903

Copyright $\odot 2021$, Academy of Managed Care Pharmacy. All rights reserved.

RESULTS: In the treatment naive analyses based on GEMINI 1 and 2, DTG/3TC dominated, i.e., was less costly and more effective, than all comparators. DTG/3TC resulted in 0.083 incremental qualityadjusted life-years (QALYs) at a cost saving of $\$ 199,166$ compared with the DTG + tenofovir disoproxil(TDF)/emtricitabine(FTC) comparator arm. The incremental QALY and cost savings for DTG/3TC compared with DTG/abacavir(ABC)/3TC, cobicistatboosted darunavir(DRV/c)/tenofovir alafenamide(TAF)/FTC, and bictegravir (BIC)/TAF/FTC, based on NMA results were $0.465,0.142$, and 0.698 , and $\$ 42,948$, $\$ 122,846$, and $\$ 44,962$, respectively. In the 
analyses of treatment-experienced virologically suppressed patients based on TANGO, DTG/3TC offered slightly lower QALYs (-0.037) with an estimated savings of $\$ 78,730$ when compared with continuation of TAF-based regimen (TBR). Sensitivity analyses demonstrated that these conclusions were relatively insensitive to alternative parameter estimates. The budget impact analysis estimated that by 5 th year a total of 70,240 treatment naive patients and 1,340,480 treatment experienced patients could be eligible to be prescribed DTG/3TC. The estimated budget savings over 5 years ranged from $\$ 1.12 \mathrm{~b}$ to $\$ 3.35 \mathrm{~b}$ (corresponding to 27,512 to 82,536 on DTG/3TC by year 5 ) in the lowest and highest uptake scenarios, respectively.

CONCLUSION: In conclusion, DTG/3TC with its comparable efficacy and lower drug acquisition costs, has the potential to offer significant cost savings to US healthcare payers for the initial treatment of treatment naive patients and as a treatment switching option for virologically suppressed patients.

Human immunodeficiency virus type 1 (HIV-1) is a retrovirus that can lead to acquired immunodeficiency syndrome (AIDS), an advanced stage of HIV infection wherein the immune system is severely damaged. Treatment for HIV infection, known as antiretroviral therapy (ART), has improved steadily since the advent of potent combination therapy in 1996. ${ }^{1}$ As ART has improved, people living with HIV (PLHIV) are able to achieve and sustain long-term viral suppression, and HIV-1 has evolved into a manageable chronic condition. ${ }^{1}$ As a result, the mean estimated duration of lifetime exposure to ART is now approximately 40 years. $^{2}$ PLHIV are surviving longer, requiring lifelong treatment, and have increased risks of chronic complications and comorbidities. ${ }^{3}$ The presence of these comorbidities in turn increases the potential for polypharmacy and drug-drug interactions (DDIs). ${ }^{4}$ Furthermore, the extended duration of ART exposure has heightened concerns about toxicities and long-term tolerability. ${ }^{5}$ All these factors have also led to increase in HIV management costs in the United States with an estimated increase of $34 \%$ in costs of HIV treatment and management of adverse events (AEs), and 176\% increase in costs of managing HIV-related comorbidities. ${ }^{6}$ While the efficacy of currently available three-drug regimens (3DRs) is well established, all classes of ART have been associated with tolerability and toxicity concerns. Simplified ART regimens with fewer components that provide equivalent efficacy with reduced ART burden should be the focus of HIV management in the future. ${ }^{7}$

Newer, more potent ARTs with higher barriers to resistance, namely dolutegravir (DTG), have now made it possible to achieve and maintain viral suppression with two antiretroviral (ARV) agents, often referred to as a 2-drug regimen. DTG/lamivudine (3TC) is one such 2-drug regimen which has been demonstrated to exhibit non-inferior efficacy and comparable safety to traditional, guidelinerecommended, 3DRs among treatment naive HIV-1 infected patients in two Phase III randomized controlled trials (GEMINI 1 and GEMINI 2). ${ }^{8}$ More recent evidence has shown that patients in these trials were able to maintain non-inferior viral suppression up to 96 weeks with no major tolerability concerns or risk of treatment emergent resistance. ${ }^{9}$ DTG/3TC has also demonstrated its noninferiority in maintaining virological suppression among patients switching to it from other guideline-recommended 3DRs in the TANGO study. ${ }^{10}$ Further, it is anticipated that DTG/3TC may reduce long term toxicities and serious DDIs by reducing ART exposure. ${ }^{11}$ DTG/3TC has also been recommended as a first line treatment for PLHIV in major guidelines. ${ }^{1,12}$ The objective of this analysis was to estimate the cost-effectiveness of DTG/3TC among naive and virologically suppressed patients initiating DTG/3TC treatment in the United States and to assess the budgetary impact of introducing DTG/3TC on payer formularies.

\section{Methods}

\section{MODEL DESIGN}

A previously published hybrid decision-tree and Markov cohort state transition mode ${ }^{13}$ was adapted to estimate the incremental costs and health outcome benefits associated with DTG/3TC compared with currently available HIV regimens among PLHIV in the United States.

Health states included in the model are based upon treatment lines, virologic response and CD4 cell count, with death as an absorbing state (Figure 1). Whilst not defined as explicit health states, patients are also subject to the risk of AIDS defining events (ADEs), treatment-related AEs, cardiovascular disease (CVD), chronic kidney disease (CKD), and fractures. A maximum of four treatment lines are included in the model: an initial ART line, two subsequent defined ART regimens, and a final unspecified salvage treatment line.

Upon model initiation, patients begin in the first ART line (first modelled line), consisting of a pairwise comparison between DTG/3TC and a comparator regimen. For each modelled arm, following discontinuation from first ART, patients are stratified amongst two possible second ART regimens, decided by their reason for discontinuation ("non-virologic" and "virologic"). Following discontinuation from the second ART, a similar stratification occurs. It is assumed that patients who discontinue due to "virologic" 


\section{FIGURE 1 Model Flow Diagram}

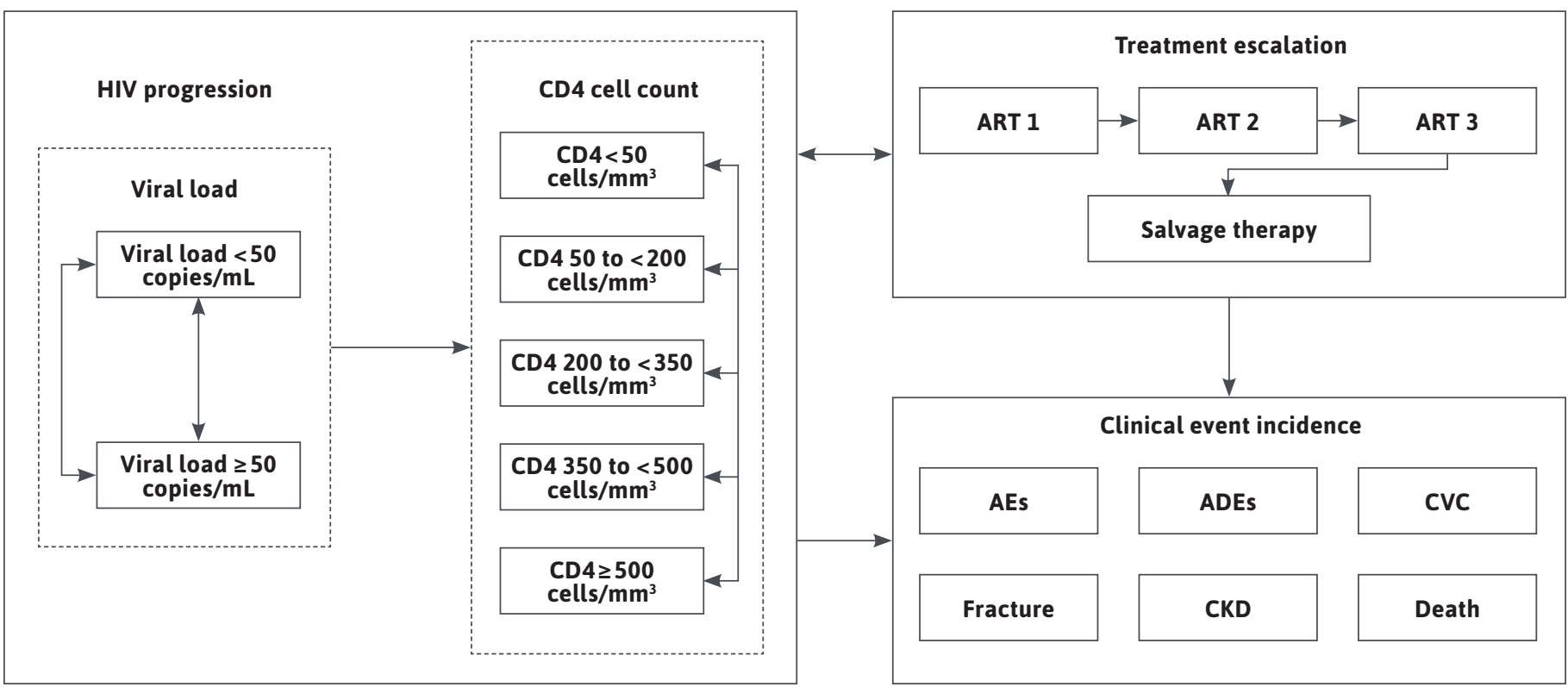

$A D E=A I D S$ defining event; $A E=$ adverse event; $A R T=$ antiretroviral therapy; $C D 4=$ cluster of differentiation $4 ; C K D=$ chronic kidney disease; $C V D=c a r d i o v a s C u l a r$ disease; HIV = human immunodeficiency virus.

reasons are likely to develop resistance, and as such have poorer suppression rates in subsequent lines of therapy. To account for differing levels of resistance at entry to the "salvage" therapy line, patients may receive one of three "salvage" therapy efficacy profiles depending on the nature of their previous reasons for discontinuation.

Upon initiation into a given treatment line, patients enter the Markov process. Consistent with previous economic models, ${ }^{14,15}$ health states included are based on viral load ( $<50$ copies $/ \mathrm{mL}, \geq 50$ copies $/ \mathrm{mL})$, CD4 cell count $(\geq 500$ cells $/ \mathrm{mm}^{3}, 350$ to $<500$ cells $/ \mathrm{mm}^{3}, 200$ to $<350$ cells $/ \mathrm{mm}^{3}$, 50 to $<200$ cells $/ \mathrm{mm}^{3},<50$ cells $/ \mathrm{mm}^{3}$ ) and death. During each cycle (one month), patients' viral load status may improve, decline, or remain constant. For a given treatment, this is represented by transitions through the health states, the rate of which is determined by treatment-specific transition matrices. In general, patients within each health state are assumed to be homogenous, except those who experience virologic failure.

Discontinuation due to virologic failure is managed by the internal decision process:

- Virologic failure: to ensure that a cohort does not indefinitely remain on a failing treatment regimen, an internal memory process identifies those who have occupied the high viral load state (>50 copies/mL) for 12 months. Patients who have failed to achieve virologic suppression within this period discontinue their current therapy. Patients discontinuing due to virologic failure remain in the same CD4 health state in the subsequent treatment arm, whilst also remaining in the high viral load state.

- Virologic rebound: patients who are achieving virologic suppression (viral load <50 copies/mL) face a monthly probability of experiencing virologic rebound. Patients experiencing virologic rebound are assigned to a higher (viral load $\geq 50$ copies $/ \mathrm{mL}$ ) viral load state in a subsequent treatment line.

- Non-virologic reasons: patients in any CD4 or viral load health state face a monthly probability of discontinuing their current line of therapy due to other non-virologic reasons. Patients who discontinue through this process remain in their existing CD4 and viral load health state.

\section{MODEL PARAMETERS}

A lifetime perspective (up to 80 years from model initiation) was adopted for the model to adequately capture all outcomes over a patients' lifetime. A cycle length of one month was set to make provision for timing of treatment switching, virologic response, CD4 cell count increases, and the 
prediction of ADEs, with the associated cost and quality of life implications. All costs and benefits were discounted at an annual rate of $3 \% .{ }^{16}$ Outcomes estimated were total costs, life-years (LYs) and quality-adjusted life-years (QALYs), with incremental cost-effectiveness ratios (ICERs) derived. The analysis was conducted from the perspective of health care payers in the United States.

Treatment Naive Patients. Two separate analyses were conducted in a treatment naive population. In the base case, DTG/3TC was compared with DTG+TDF/FTC using estimates obtained from the GEMINI trials. ${ }^{8,9}$ In the scenario analyses, DTG/3TC was compared with the most commonly used guideline recommended 3DRs in the United States, bictegravir (BIC)/TAF/FTC (Biktarvy), DTG/ $\mathrm{ABC} / 3 \mathrm{TC}$ (Triumeq) and DRV/c/TAF/FTC (Symtuza) via use of a published network meta-analysis (NMA) comparing these regimens to DTG/3TC. ${ }^{17,18}$ Second and third lines were represented by a pooled comparator where the cost was an average of 16 different ART regimens utilized in current US practice. Estimates of virologic suppression and CD4 cell counts for stable patients were taken from studies by Antinori and Baril, respectively, whilst those for failing population were estimated from Kanters study. ${ }^{19-21}$

Treatment Experienced Virologically Suppressed Patients. In treated patients who were virologically suppressed on a first-line regimen, DTG/3TC was compared with continuing a TAF-based 3 or 4 drug regimen (cTBR). Efficacy estimates were obtained from the TANGO trial which evaluated the efficacy and safety of switching to DTG/3TC compared with cTBR. ${ }^{10}$ Second- and third-line treatments were represented by a pooled comparator, similar to treatment naive analyses.

\section{CLINICAL DATA}

Patient demographic and clinical data at baseline was obtained from GEMINI 1 and 2 in treatment naive analyses and from TANGO in treatment experienced, virologically suppressed patients (Supplementary Table 1, available in online article). ${ }^{8,10}$ Model parameters not reported in clinical trials were obtained from published literature or expert consultation.

ART efficacy, estimated as virologic response, was defined as the achievement of a viral load (HIV-1 RNA) $<50$ copies/mL, and immunological response, defined as the average increase in CD4 cell count. These measurements informed transition matrices that were used in the model to control patients' movement between viral load and CD4 cell count based health states. Data from 0-48 weeks was used to inform this time period and data from 48-96 weeks was used to inform all subsequent 48 -week periods (including post 96 weeks). The assumption therefore was that the pattern of observed movement from 48-96 weeks is consistent as long as a patient remains in the first line treatment. Efficacy parameters used in the model are displayed in Table 1. Transition matrices and a further description of their derivation (Supplementary Tables 2-37, available in online article).

Mortality in the model included all-cause and HIVrelated death, and mortality due to ADEs and CVD. Rates of all-cause mortality were estimated using US genderspecific life tables. ${ }^{26}$ To reflect the additional mortality in the HIV-1 population, published estimates of relative risks comparing mortality in the HIV-1 population to that of the general population are included in the model. ${ }^{27}$ Patients experiencing ADEs also faced increased risks of mortality. This additional risk was estimated from the literature and applied additively to the all-cause mortality risk..$^{28}$ The model did not include any adjustment for CVD-related mortality, assumed to be incorporated within the CD4 health state relative risks.

The model included five categories of ADEs. Acute viral opportunistic infections (OI) included cytomegalovirus; acute bacterial OIs included mycobacterium avium infection and tuberculosis; acute fungal OIs included pneumocystis carinii pneumonia, cryptococcosis, and esophageal candidiasis; and acute protozoal infections included cerebral toxoplasmosis. Other ADEs included lymphoma, HIV-1 wasting syndrome, HIV-1 dementia, and Kaposi's sarcoma. The probabilities of occurrence of specific ADEs were associated with CD4 cell count and time on treatment and were estimated from the literature. ${ }^{29}$ The incidence of ADEs in turn influences mortality, disease management costs, and quality of life. ADEs were modelled by the probability of occurrence within particular health states; if patients experienced them there were related mortality, utility and cost outcomes (Supplementary Table 39, available in online article).

Health related quality of life was estimated using CD4+ health state-based utilities calculated using patient level data from the GEMINI 1 and 2 trials for treatment naive analyses and from the TANGO study for treatment experienced analyses. In the TANGO trial, there were no patients with a CD4 cell count below 50 and therefore it was not possible to observe the utility value for these patients. It is conservatively assumed that these patients have the same utility as those who have a CD4 cell count under 200. As there are no patients starting in this health state, it is not assumed to affect results substantially. Utility decrements were applied for the presence of CVD and CKD, and the occurrence of fractures, AEs and ADEs. CVD events and fractures had an initial event-related utility decrement followed by chronic disutility whilst CKD, AEs and ADEs had 


\section{TABLE 1 Efficacy Parameters for Available Efficacy Profiles}

\begin{tabular}{|c|c|c|c|c|c|c|}
\hline \multirow[b]{3}{*}{ Efficacy profile } & \multirow[b]{3}{*}{ Timepoint } & \multirow[b]{2}{*}{$\begin{array}{c}\text { Virologic } \\
\text { suppression }\end{array}$} & \multirow[b]{2}{*}{$\begin{array}{c}\text { Baseline } \\
\text { CD4 cell count }\end{array}$} & \multirow[b]{2}{*}{$\begin{array}{l}\text { Change in } \\
\text { CD4 cell count }\end{array}$} & \multicolumn{2}{|c|}{ Monthly discontinuation } \\
\hline & & & & & $\begin{array}{c}\text { Virologic } \\
\text { discontinuation }\end{array}$ & $\begin{array}{c}\text { Non-virologic } \\
\text { discontinuation }\end{array}$ \\
\hline & & Mean (SE), \% & Mean (SD) & Mean (SD) & \multicolumn{2}{|c|}{ Mean (SE) } \\
\hline \multirow[t]{2}{*}{ DTG/3TC (naive) ${ }^{8}$} & Wk 0-48 & $91.48(1.04)$ & $462.00(219.3)$ & $224.10(172.2)$ & $0.0023(0.0018)$ & $0.0056(0.0028)$ \\
\hline & Wk 48-96 & 86.03 (1.04) & $687.90(270.7)$ & $44.90 \quad(16.5)$ & $0.0003(0.0006)$ & $0.0045(0.0025)$ \\
\hline \multirow[t]{2}{*}{ DTG+TDF/FTC ${ }^{8}$} & Wk 0-48 & $93.31(0.93)$ & $462.00(219.3)$ & $217.10(190.6)$ & $0.0015(0.0015)$ & $0.0047(0.0025)$ \\
\hline & Wk 48-96 & $89.53(0.93)$ & $682.60(292.0)$ & $40.60 \quad(17.4)$ & $0.0001(0.0004)$ & $0.0032(0.0021)$ \\
\hline \multirow[t]{2}{*}{$\mathrm{DTG} / \mathrm{ABC} / 3 \mathrm{TC}^{17}$} & Wk 0-48 & 91.28 (1.05) & $462.00(219.3)$ & $254.20(172.2)$ & $0.0025(0.0018)$ & $0.0047(0.0025)$ \\
\hline & Wk 48-96 & $86.03(1.05)$ & $716.14(270.7)$ & $-16.80 \quad(16.5)$ & $0.0019(0.0012)$ & $0.0051(0.0019)$ \\
\hline \multirow[t]{2}{*}{$\mathrm{DRV} / \mathrm{c} / \mathrm{TAF} / \mathrm{FTC}^{\mathrm{a}, 17}$} & Wk 0-48 & 84.38 (1.36) & $462.00(219.3)$ & $216.60(172.2)$ & $0.0091(0.0034)$ & $0.0047(0.0025)$ \\
\hline & Wk 48-96 & $68.03(1.03)$ & $678.58(270.7)$ & NA & $0.0019(0.0012)$ & $0.0051(0.0019)$ \\
\hline \multirow[t]{2}{*}{$\mathrm{BIC} / \mathrm{TAF} / \mathrm{FTC}^{17}$} & Wk 0-48 & 90.58 (1.09) & $462.00(219.3)$ & $258.80(172.2)$ & $0.0031(0.0021)$ & $0.0047(0.0025)$ \\
\hline & Wk 48-96 & $86.03(1.05)$ & $720.76(270.7)$ & $-32.1 \quad(16.5)$ & $0.0019(0.0012)$ & $0.0051(0.0019)$ \\
\hline DTG/3TC (experienced) $)^{10}$ & Wk 0-48 & $93.22(1.31)$ & $702.00(289.2)$ & $29.20(179.5)$ & $0.0002(0.0008)$ & $0.0061(0.0040)$ \\
\hline $\mathrm{CTBR}^{10}$ & Wk $0-48$ & $93.01(1.31)$ & $726.00(273.5)$ & $2.90(178.6)$ & $0.0005(0.0011)$ & $0.0060(0.0040)$ \\
\hline Treatment experienced: stable switch $\mathrm{h}^{\mathrm{b}, 19,20}$ & All periods & $88.98(1.26)$ & $540.00(232.5)$ & $69.25(149.1)$ & $0.0053(0.0003)$ & $0.0079(0.0004)$ \\
\hline Treatment experienced: failing switchc,21 & All periods & 73.78 (3.69) & $168.70(155.1)$ & $176.40(149.3)$ & $0.0165(0.0008)$ & $0.0024(0.0001)$ \\
\hline Salvage $1^{\mathrm{d}, 22,23}$ & All periods & $71.04(3.55)$ & $151.00(141.0)$ & $119.00(132.3)$ & NA & NA \\
\hline Salvage $2^{\mathrm{d}, 24,25}$ & All periods & $60.60(3.03)$ & $151.00(141.0)$ & $111.00(146.3)$ & NA & NA \\
\hline Salvage $3^{\mathrm{d}, 24,25}$ & All periods & $50.80(2.54)$ & $151.00(141.0)$ & $71.00(100.8)$ & NA & NA \\
\hline
\end{tabular}

aThe efficacy profile assigned to the DRV/C/TAF/FTC profile is informed by DRV/r/TDF/FTC where cobicistat-boosted darunavir is assumed equally efficacious as ritonavir-boosted darunavir and TAF/FTC is assumed equally efficacious to TDF/FTC

${ }^{b}$ Virologic suppression taken from Antinori et al.; weighted mean of included studies reporting variable of interest in Baril et al used for CD4 cell count

"Weighted mean of included studies reporting variable of interest

${ }^{d} B a s e d$ on the raltegravir arm of the clinical trial

$3 T C=$ lamivudine $: A B C=$ abacavir $: B I C=$ bictegravir $: C D 4=$ cluster of differentiation $4 ; C T B R=$ continuation of $T A F-$ based regimen: $D R V / C=$ cobicistat-boosted darunavir; DTG = dolutegravir; FTC=emtricitabine; $N A=$ not applicable; $T A F=$ tenofovir alafenamide; $T D F=$ tenofovir disoproxil fumarate; $S D=s t a n d a r d$ deviation; $\mathrm{SE}=$ standard error; $\mathrm{Wk}=$ week

an associated chronic utility decrement (Supplementary Table 38, available in online article). An age dependent utility decrement was also applied additively to all patients. ${ }^{30}$

Long-term toxicities associated with TDF/FTC were included with the impact on cholesterol, bone mineral density (BMD) and estimated glomerular filtration rate (eGFR) in line with observations in GEMINI trials. ${ }^{89}$ Outcomes modelled were renal decline, CVD and fracture incidence. The age -and cholesterol- dependent probability of developing CVD was estimated with the Framingham risk equation; ${ }^{31}$ fracture incidence was estimated using data from a study by Kanis et $\mathrm{al}^{32}$ and renal decline was estimated based on a study by Gianotti et al. ${ }^{33}$ This effect of treatment on cholesterol, BMD and eGFR was applied where the intervention included a TDF/FTC-based regimen whilst for all other interventions, a DTG/3TC-based profile was used. This assumption was used for both treatment naive and treatment experienced analyses.

\section{COSTS}

Drug acquisition costs were obtained from MDDB US product price reporting system ${ }^{34}$ drug costs reflect wholesale acquisition costs (WAC). The monthly cost of DTG/3TC was estimated to be $\$ 2,408$, whilst the costs of DTG + TDF/FTC, DTG/ABC/3TC, DRV/c/TAF/FTC, BIC/TAF/FTC, cTBR and a pooled comparator were estimated to be $\$ 3,669, \$ 3,032$, $\$ 3,722, \$ 3,238, \$ 3,103$, and $\$ 3,254$, respectively. The monthly cost for salvage therapy, assumed to be treatment with ritonavir-boosted darunavir (DRV/r)/TAF/FTC, was estimated to be $\$ 3,722.35$ 
Costs associated with the management of AEs were applied as per event costs in the cycle of $\mathrm{AE}$ incidence. Costs associated with common drug-related AEs (incidence $\geq 1 \%$ ) were estimated from literature ${ }^{36}$ and inflated costs were applied to AE rates derived from GEMINI and TANGO for treatment naive and treatment experienced analyses, respectively. The resultant costs included in the model were \$277 (Standard Error [SE]: \$28) for nausea and dizziness, and \$214 (SE: \$21) for diarrhea, soft feces, or flatulence. Other AEs were assumed to incur no additional costs.

Additional costs included in the model were those associated with outpatient care, ADEs, non-HIV medications, management of CVD, and end of life care. Resource use associated with all-cause health encounters was expected to vary significantly between CD4 cell count health states and as such was estimated for each CD4 health state using data from publications of the HIV Research Network as reported in a study by Farnham and colleagues (Supplementary Table 38, available in online article). ${ }^{37}$ The costs associated with ADEs, CVD, CKD, fractures, and end of life care were derived from published literature (Supplementary Table 38). ${ }^{8,10,35,37,53-55}$ The cost of CVD was assumed to incur an initial event cost followed by a monthly disease management cost. As the mean eGFR of the cohort changed over time, patients were assumed to transition through progressively worsening CKD categories. Each CKD category had an associated cost which was applied as a function of the time spent in that category. Costs of stage $1 \mathrm{CKD}$ were assumed to be no more than the general population and were set to zero.

All costs are expressed in 2019 US dollars and were inflated to 2019 where needed. ${ }^{38}$ An annual discount rate of $3 \%$ was used for costs and outcomes as per Institute for Clinical and Economic Review guidelines. ${ }^{16}$

\section{MODEL VALIDATION}

The model was internally validated with independent double programming of the visual basic functions. It was also reviewed by a third researcher to ensure accuracy. External validation was undertaken using previously published costeffectiveness studies that utilized similar models. ${ }^{24,39-42}$ For each validation exercise, model inputs corresponding to published profiles were run within the DTG/3TC costeffectiveness model. Where model inputs were not reported, default model inputs were used, or reasonable assumptions were made. The predicted values for total costs, LYs and QALYs were compared with published values. Consistent with previously published health economic validation studies, goodness of fit was measured using the coefficient of determination $\left(\mathrm{R}^{2}\right)$, mean absolute percentage error (MAPE) and the root mean square percentage error (RMSPE).

\section{COST-EFFECTIVENESS ANALYSIS}

In the deterministic analyses, DTG/3TC was compared with DTG + TDF/FTC, DTG/ABC/3TC, DRV/c/TAF/FTC and BIC/ TAF/FTC using mean values as inputs for each of the model parameters. Univariate sensitivity analysis was undertaken by varying model parameters across pre-defined ranges. These ranges for patient baseline characteristics and model settings are displayed in Table 1. Other parameters such as virological suppression up to week 96, virologic and nonvirologic discontinuations, mortality, health state utilities, DTG/3TC drug acquisition cost, ADE costs and health state costs were varied by $20 \%$. A probabilistic sensitivity analysis (PSA) was also performed by varying each parameter simultaneously within their respective distributions.

\section{BUDGET IMPACT ANALYSIS}

Further, a 5-year budget impact analysis was conducted of implementing DTG/3TC treatment strategy among treatment naive and treatment experienced, virologically suppressed PLHIV in the United States to estimate the potential cost savings (further details provided in the Supplementary Materials). This analysis was designed as an update to an earlier study by Girouard et al $2015 .{ }^{43}$ This modelling exercise sought to evaluate the budget impact of the introduction of dual therapy (DTG and 3TC) but was conducted prior to the results of the clinical trials evaluating DTG/3TC. Here, we sought to provide an update to this analysis in light of the non-inferior trial results and details of the cost of the regimen.

Patients eligible to receive DTG/3TC were estimated from United States epidemiological data. ${ }^{4}$ Twenty percent of the Centers for Disease Control and Prevention estimated 1.2M people living with HIV (reflecting currently suppressed patients who have never experienced virologic failure) and $37 \%$ of the approximately 38,000 newly diagnosed each year were expected to be eligible to switch to DTG/3TC, consistent with the earlier study by Girouard et al. ${ }^{43}$ Of these eligible patients, three scenarios of DTG/3TC use were evaluated as follows: (1) $5 \%$ naive/10\% experienced, (2) $10 \%$ naive/20\% experienced, and (3) $15 \%$ naive/30\% experienced. Uptake of DTG/3TC was assumed to be taken proportionally from existing HIV drug regimens based on their current market share in the United States. The current market shares for Integrase Strand Inhibitors (INSTIs), Non-Nucleoside Reverse Transcriptase Inhibitors (NNRTIs), Protease Inhibitors (PIs), and others were estimated to be $66 \%, 13 \%, 8 \%$, and $12 \%$; changing to $71 \%, 9 \%, 8 \%$, and $12 \%$ over next 5 years, respectively, in the absence of DTG/3TC. The average cost for an INSTI core-agent-based treatment was calculated to be $\$ 3,265 /$ month, estimated using the market shares of individual INSTI-based treatments. 


\section{FIGURE 2 Cost Effectiveness Scatter Plot for DTG/3TC Compared to DTG + TDF/FTC in Treatment Naive PLHIV}

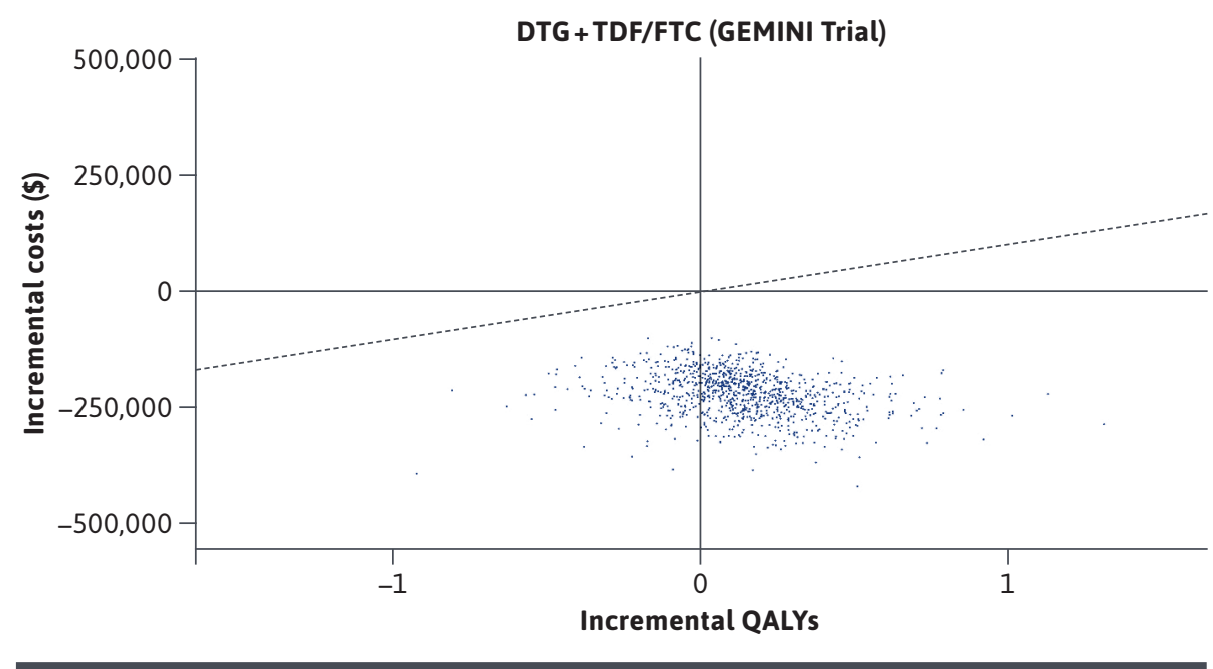

The corresponding monthly cost for NNRTIs-based, PI-based and other ARTs was $\$ 2,445, \$ 3,676$, and $\$ 2,513$, respectively. The cost of DTG/3TC (\$2,408 per month) is lower than the average regimen cost of the most widely used drug class, INSTI coreagent-based treatment, at \$857 less per month (\$10,287 less per year). The total cost of the scenarios with and without the addition of DTG/3TC were compared to evaluate the budget impact of the introduction of DTG/3TC.

\section{Results}

In the treatment naive analysis estimated at 3\% discounting, DTG/3TC was associated with 18.064 QALYs and 20.126 LYs, compared with 17.981 QALYs and 20.146 LYs in the DTG + TDF/FTC arm. The estimated total lifetime costs for DTG/3TC and DTG+TDF/FTC were $\$ 1,236,776$, and $\$ 1,435,942$, respectively. Resultantly, DTG/3TC dominated DTG + TDF/FTC, albeit with a very small incremental QALY benefit. Univariate sensitivity analysis indicated that proportion of patients experiencing viral suppression and treatment specific changes in eGFR were the most influential parameters in treatment naive analyses (Supplementary Figure 1, available in online article). The corresponding parameters in treatment experienced analysis were probability of non-virologic discontinuation for DTG/3TC and the comparators (Supplementary Figure 2, available in online article). Results of the PSA were consistent with DTG/3TC dominating DTG + TDF/FTC in $76 \%$ of iterations (Figure 2) and cost saving in all iterations. When comTAF/FTC, BIC+TAF/FTC, using NMA results, DTG/3TC was estimated to result in lower total costs compared to all three comparators (Table 2). DTG/3TC dominated all the three comparators, with a greater number of QALYs versus all comparators. The PSA results showed DTG/3TC to dominate DTG/ABC/3TC, DRV/c/ TAF/FTC, and BIC+TAF/FTC in $87 \%$, $88 \%$, and $87 \%$ simulations, respectively. Among treatment experienced, pared with DTG/ABC/3TC, DRV/c/ virologically suppressed patients DTG/3TC resulted in lower QALYs (12.586 vs 12.623) and lower costs $(\$ 3,021,650$ vs $\$ 3,100,380)$ compared to cTBR (Table 2). DTG/3TC offering near-equivalent QALYs while saving an estimated $\$ 78,730$ indicated an ICER of $\$ 2.1 \mathrm{M}$ when TBRs are used instead of DTG/3TC. PSA results indicated 37\% simulations wherein DTG/3TC would result in higher QALYs and lower costs, thereby dominating cTBR (Figure 3). A further scenario analysis was conducted by shortening the model time horizon to two years. Results in the treatment naive settings suggested that patients treated with DTG/3TC resulted in near-identical QALYs at a saving of $\$ 25,933$ compared with DTG + TDF/FTC. Against other comparators, QALYs were similar with cost savings ranging from $\$ 12,457$ against DTG/ABC/3TC to $\$ 25,859$ against DRV/c/TAF/FTC. Similarly, DTG/3TC was also cost savings $(\$ 14,847)$ with near-identical QALYs in treatment experienced patients.

The model exhibited a high degree of consistency with previously published cost-effectiveness analyses, with RMSPE and MAPE values of $14.7 \%$ and $17.7 \%$, respectively. The overall $\mathrm{R}^{2}$ value was 0.937 suggesting a high degree of correlation between observed and predicted endpoints. The results of validation exercise presented as normalized observed (model predicted) versus expected (published results) results for costs, life years (LYs) and quality adjusted life years (QALYs) are presented in Supplementary Figure 3 (available in online article).

In the budget impact analysis, the expected number of treatment naive patients initiating DTG/3TC ranged from 702 in scenario 1 to 2,107 in scenario 3 . The corresponding number of treatment experienced patients switching to DTG/3TC by the end of 5 years ranged from 26,810 in scenario 1 


\begin{tabular}{|c|c|c|c|c|c|c|c|c|}
\hline Treatment & Total QALYs & Total LYs & $\begin{array}{c}\text { Total costs } \\
\$\end{array}$ & $\begin{array}{c}\text { Incidence of } \\
\text { AEs }\end{array}$ & $\begin{array}{c}\text { Incidence of } \\
\text { ADEs }\end{array}$ & $\begin{array}{c}\text { Incidence of } \\
\text { CVD }\end{array}$ & $\begin{array}{l}\text { Incidence of } \\
\text { fractures }\end{array}$ & $\begin{array}{c}\text { ICERs of } \\
\text { DTG/3TC vs } \\
\text { comparator } \\
\text { (cost/QALY, \$) }\end{array}$ \\
\hline \multicolumn{9}{|l|}{ Treatment naive } \\
\hline DTG/3TC & 18.064 & 20.126 & $1,236,776$ & 1.049 & 0.117 & 49.52 & 0.144 & - \\
\hline DTG + TDF/FTC & 17.981 & 20.146 & $1,435,942$ & 1.792 & 0.118 & 53.95 & 0.150 & Dominant $^{a}$ \\
\hline Incremental & 0.083 & -0.020 & $-199,166$ & -0.743 & -0.001 & -4.43 & -0.007 & \\
\hline DTG/3TC & 18.064 & 20.126 & $1,236,776$ & 1.049 & 0.117 & 49.52 & 0.144 & - \\
\hline $\mathrm{BIC}+\mathrm{TAF} / \mathrm{FTC}$ & 17.366 & 19.314 & $1,281,737.95$ & 0.686 & 0.135 & 45.58 & 0.132 & Dominant $^{\mathrm{a}}$ \\
\hline Incremental & 0.698 & 0.812 & $-44,961.75$ & 0.363 & -0.018 & 3.95 & 0.012 & \\
\hline DTG/ABC/3TC & 17.599 & 19.581 & $1,279,724.51$ & 1.212 & 0.128 & 46.77 & 0.135 & Dominant $^{\mathrm{a}}$ \\
\hline Incremental & 0.465 & 0.545 & $-42,948.31$ & -0.163 & -0.011 & 2.76 & 0.009 & \\
\hline $\mathrm{DRV} / \mathrm{c} / \mathrm{TAF} / \mathrm{FTC}$ & 17.921 & 19.944 & $1,359,622.51$ & 4.094 & 0.118 & 48.18 & 0.139 & Dominant $^{a}$ \\
\hline Incremental & 0.142 & 0.182 & $-122,846.31$ & -3.045 & -0.001 & 1.34 & 0.005 & \\
\hline \multicolumn{9}{|c|}{ Treatment experienced } \\
\hline DTG/3TC & 12.586 & 17.553 & $3,021,650$ & 0.598 & 0.095 & 81.42 & 0.125 & - \\
\hline CTBR & 12.623 & 17.606 & $3,100,380$ & 0.092 & 0.093 & 81.70 & 0.126 & $2,153,802^{b}$ \\
\hline Incremental & -0.037 & -0.053 & $-78,730$ & 0.506 & 0.001 & -0.28 & 0.000 & \\
\hline
\end{tabular}

${ }^{a}$ Dominant - Intervention is more effective and less costly than the comparator

${ }^{b} \mathrm{DTG} / 3 \mathrm{TC}$ cost-effective compared to cTBR

$3 T C=$ lamivudine; $A B C=$ abacavir; $A E=$ adverse event; $A D E=A I D S$ defining event; $B I C=$ bictegravir; $C V D=$ cardiovascular disease; $c T B R=$ continuation of $T A F$ based regimen; $D T G=$ dolutegravir; $D R V / C=$ cobicistat boosted darunavir; $F T C=$ emtricitabine; $I C E R=$ incremental cost-effectiveness ratio; $L Y=l i f e$ year; $T A F=$ tenofovir alafenamide fumarate; TDF = tenofovir disoproxil fumarate; $Q A L Y=$ quality adjusted life year.

to 80,429 in scenario 3 . The resulting cost saving associated with introduction of DTG/3TC in year 1 ranged from $\$ 206 \mathrm{M}$ in scenario 1 to $\$ 618 \mathrm{M}$ in scenario 3 (Table 3). Overall, introduction of DTG/3TC was estimated to result in significant healthcare savings over 5 years ranging from $\$ 1.12 \mathrm{~B}$ to $\$ 3.35 \mathrm{~B}$ in the scenario 1 and scenario 3, respectively. For every $0.5 \%$ and $1 \%$ increase in the use of DTG/3TC in naive and experienced patients, there was an estimated $\$ 112 \mathrm{M}$ in further savings over 5 years.

\section{Discussion}

The fixed-dose 2-drug regimen DTG/3TC offers an alternative to traditional 3DRs, offering the potential to reduce lifelong drug exposure and costs without compromising clinical efficacy or safety for treatment naive and treatment experienced virologically suppressed PLHIV, $, 8,90$ and has been recommended as an initial therapy in major clinical treatment guidelines. ${ }^{1,12}$ In this analysis, the cost-effectiveness of DTG/3TC as a maintenance treatment for PLHIV was evaluated. In the treatment naive PLHIV, DTG/3TC resulted in comparable QALYs with substantially lower costs to other standard of care (SoC) ART regimens. This is consistent with the non-inferiority results obtained in GEMINI 1 and 2 wherein participants treated with DTG/3TC achieved comparable viral suppression to those treated with a DTG+TDF/FTC based 3DR. Further, DTG/3TC was compared with guideline recommended, SoC fixed-dose combinations (FDC) such as DTG/ABC/3TC, DRV/c/TAF/ FTC, and BIC/TAF/FTC. A published study has reported comparable efficacy and safety of DTG/3TC to these 3 -drug FDCs in treatment naive PLHIV,${ }^{17}$ and our analyses demonstrated its dominance in an economic context. The results in treatment experienced patients were similar, demonstrating treatment with DTG/3TC is likely to broadly maintain outcomes at lower costs among virologically suppressed patients switching to DTG/3TC. In this analysis, should TAF-based regimens be used instead of DTG/3TC, the estimated incremental cost-per-QALY is $>\$ 2 \mathrm{M}$. This far exceeds willingness to pay thresholds widely considered to be cost-effective in the United States (eg ICER $\$ 100,000$ per QALY gained). Furthermore, building on the results from the 


\section{FIGURE 3 Cost-Effectiveness Scatter Plot for DTG/3TC Compared to CTBR in Treatment Experienced Virologically Suppressed PLHIV}

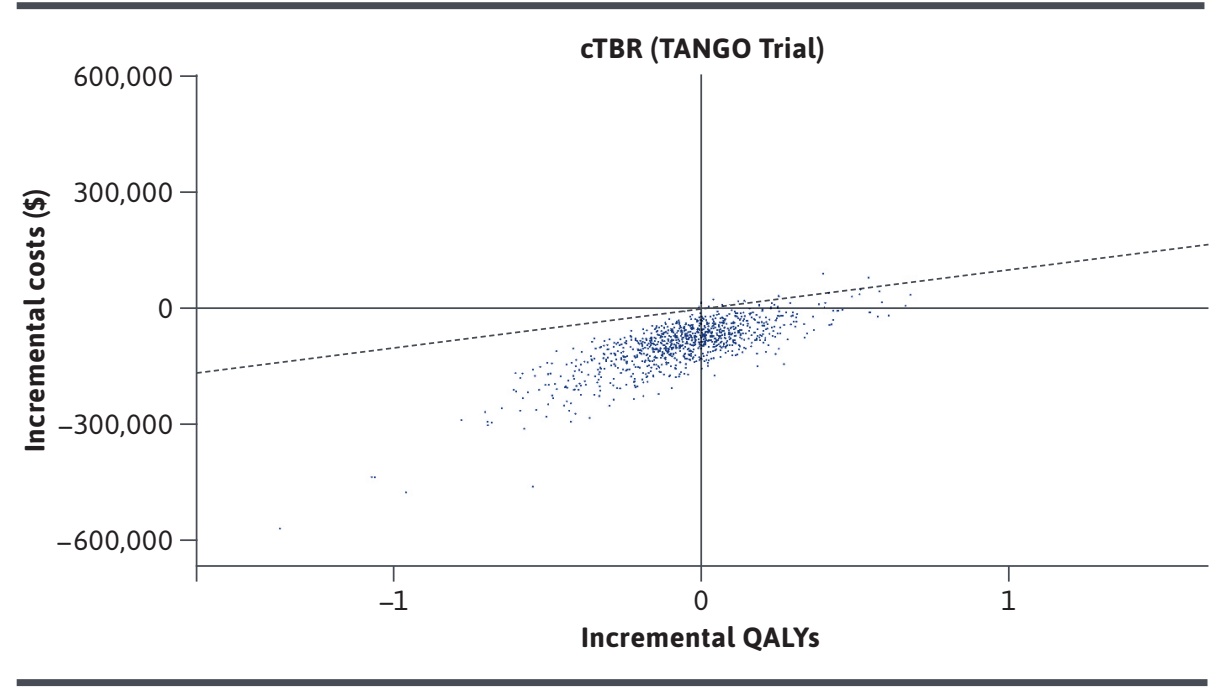

cost-effectiveness analysis, the budget impact estimates demonstrated potential for substantial cost-savings, which were robust in all scenarios. DTG/3TC FDC may therefore present a valuable opportunity for obtaining significant budgetary savings without compromising treatment efficacy or safety.

A study by Girouard et al, conducted and published prior to data availability from the GEMINI studies, hypothesized significant cost savings with DTG+3TC. ${ }^{44}$ The authors compared DTG+3TC with DTG/ABC/3TC, the SoC at the time of the study, and reported DTG +3TC to generate slightly fewer QALYs at significantly lower costs compared to SoC. They forecasted savings up to $\$ 3.4 \mathrm{~B}$ if $50 \%$ of naive patients and $25 \%$ of eligible experienced patients switched to DTG/3TC over 5 years. As their analysis pre-dated the availability of head to head trial results, Girouard and colleagues made some simplifying assumptions in their model inputs compared to 3DRs. They assumed that
DTG + 3TC would be slightly less effective at achieving virologic suppression and would be more likely to experience virologic failure over 96 weeks. As previously indicated, results from the two GEMINI studies and from the TANGO study demonstrated comparable and durable virologic suppression of DTG/3TC compared to SoC 3DRs. ${ }^{8,9,10}$ Additionally, Girouard and colleagues assumed no adherence benefits to co-formulated single tablet regimens (STRs) compared to multitablet regimens based on clinical trial experience but these assumptions have not borne out in real world settings where patients are not highly managed to strict protocol visits and procedures. The body of real-world observational evidence continues to grow suggesting adherence benefits to STR formulations in real world settings and that high adherence is associated with improved clinical outcomes and reduced costs. ${ }^{45-51}$ Using a similar approach to calculate the budgetary impact of DTG/3TC to Girouard and colleagues, but with model assumptions updated to reflect current clinical trials results and published WAC prices for DTG/3TC and other SoC STRs, our results were broadly consistent with their predictions with costs savings of $\$ 3.3 \mathrm{~B}$ attributable to $15 \%$ eligible naive and $30 \%$ eligible experienced patients switching therapy. Whilst the precise proportions of naive and switch differ between the two studies, the overall patient numbers are comparable.

Certain considerations should be acknowledged when interpreting the findings of this analysis, perhaps warranting the need for further research. As the life expectancy of PLHIV increases, so does their exposure to ART therapy for long periods of time. This may increase the likelihood of DDIs alongside the incidence of ARV-related long-term toxicities, such as CVD, renal impairment, and bone fractures. ${ }^{6}$ Two-drug regimens such as DTG/3TC have the potential to alleviate the negative impact of long-term ARV therapy. Our analysis considered this benefit to the extent it was reported in the GEMINI trials, but further research is needed to estimate the true impact due to a relative paucity of long-term data. Further modelling, supplemented by real-life data, is needed to estimate the impact of DTG/3TC over a patients' lifetime. Our analysis was based on 96-week data from GEMINI 1 and 2 and an NMA within naive PLHIV. Longterm outcomes in the model were based on observed trial results. Such extrapolation comes with its inherent uncertainty, further compounded by the non-inferior nature of the treatment comparison and, consequently, small differences in observed efficacy. To mitigate this uncertainty, the model was extensively validated internally and externally, and a scenario analysis with shorter time horizon was conducted, which showed consistent results. 


\begin{tabular}{|c|c|c|c|c|c|}
\hline $\begin{array}{l}\text { Market share } \\
\text { scenarios }\end{array}$ & Year 1 & Year 2 & Year 3 & Year 4 & Year 5 \\
\hline \multicolumn{6}{|c|}{ Number of treatment experienced patients on DTG/3TC } \\
\hline Scenario 1 & 24,000 & 24,702 & 25,405 & 26,107 & 26,810 \\
\hline Scenario 2 & 48,000 & 49,405 & 50,810 & 52,214 & 53,619 \\
\hline Scenario 3 & 72,000 & 74,107 & 76,214 & 78,322 & 80,429 \\
\hline \multicolumn{6}{|c|}{ Number of treatment naive patients on DTG/3TC } \\
\hline Scenario 1 & 702 & 702 & 702 & 702 & 702 \\
\hline Scenario 2 & 1,405 & 1405 & 1,405 & 1,405 & 1,405 \\
\hline Scenario 3 & 2,107 & 2,107 & 2,107 & 2,107 & 2,107 \\
\hline \multicolumn{6}{|c|}{ Annual cost savings with the introduction of DTG/3TC ( $\$$ millions) } \\
\hline Scenario 1 & 206 & 216 & 224 & 232 & 240 \\
\hline Scenario 2 & 412 & 431 & 447 & 463 & 479 \\
\hline Scenario 3 & 618 & 647 & 671 & 695 & 719 \\
\hline \multicolumn{6}{|c|}{ Cumulative cost savings with the introduction of DTG/3TC ( $\$$ millions) } \\
\hline Scenario 1 & 206 & 422 & 645 & 877 & 1116 \\
\hline Scenario 2 & 412 & 843 & 1,290 & 1,753 & 2,233 \\
\hline Scenario 3 & 618 & 1,265 & 1,935 & 2,630 & 3,349 \\
\hline \multicolumn{6}{|c|}{$\begin{array}{l}\text { Scenario 1: DTG/3TC market share of } 5 \% \text { in treatment naive and } 10 \% \text { in treatment experienced; } \\
\text { Scenario 2: DTG/3TC market share of } 10 \% \text { in treatment naive and } 20 \% \text { in treatment experienced; } \\
\text { Scenario 3: DTG/3TC market share of } 15 \% \text { in treatment naive and 30\% in treatment experienced } \\
\text { 3TC= lamivudine; DTG = dolutegravir. }\end{array}$} \\
\hline
\end{tabular}

Since the primary aim of economic evaluation of HIV technologies is to inform health care decision making, in this respect the time horizon for economic evaluations should be sufficiently long enough to capture all the potential costs and benefits associated with a new technology under consideration at the identified comparators. Guidelines for economic evaluations confirm this recommendation and also state that justification for the economic evaluation time horizon should be clearly stated. This distinguishes economic evaluation from clinical efficacy evaluation, which is based only on the follow-up period stated within the trial or group of trials which is similarly justified in the clinical trial protocol(s). The sequential nature of HIV technologies specifies that the introduction of a new technology to the sequence of treatment regiments clearly impacts on the subsequent treatment regimens that are included in the sequence. Hence, for this reason, the majority of HIV economic evaluations take a lifetime time horizon, which has been identified as the most common time horizon in SLRs of HIV treatment economic evaluations. Caution should be taken when considering shorter time horizons for HIV technologies, in that the entirety of the expected costs and benefits associated with the intervention and comparator would not be captured in the analysis and hence could lead to ill-informed decision-making inferences. Concerns about the evidence synthesis nature of model-based economic evaluation and extrapolation of single RCT data would not be address by considering shorter model time horizons. However, shorter model time horizons may help with model validation exercises to inform on how well the model performs with a view to the RCT outputs.

We did not include adherence as a parameter in the model. Whilst adherence is important among PLHIV, the treatments being compared were oral daily ARTs, expected to have comparable adherence in real world clinical setting. Differences, if any, were likely to favor DTG/3TC which is a single tablet regimen compared to several multiple tablet regimen comparators.

\section{Conclusion}

In conclusion, DTG/3TC is the first globally approved 2-drug ARV regimen (Food and Drug Administration (FDA)/ European Medicines Agency (EMA)) for treatment-naive PLHIV. It has the potential to deliver benefits associated with reduced ART exposure, reduced long-term toxicity. With its comparable efficacy, durability, and lower drug acquisition costs, DTG/3TC has the potential to offer significant cost benefits to healthcare payers in the United States.

\section{DISCLOSURES}

This study was funded in full by ViiV healthcare, Brentford, UK. Medical writing to support this study was also funded in full by ViiV Healthcare, Brentford, UK. Butler, Hayward, and Jacob are employees of HEOR Ltd, the company performing this study funded by ViiV Healthcare. Anderson is an employee of GlaxoSmithKline and owns shares in the company. Punekar, Evitt, and Oglesby are employees of ViiV Healthcare and own stocks in GlaxoSmithKline. 


\section{REFERENCES}

1. Panel on Antiretroviral Guidelines for Adults and Adolescents. Guidelines for the Use of Antiretroviral Agents in Adults and Adolescents with HIV. Department of Health and Human Services. Accessed 03 April 2020. https://clinicalinfo.hiv.gov/ en/guidelines

2. Nakagawa F, Lodwick RK, Smith CJ, et al. Projected life expectancy of people with HIV according to timing of diagnosis. AIDS. 2012;26(3):335-43.

3. Chronic comorbidities and coinfections in PLHIV. World Health Organization. Accessed April 3, 2020. http://www.who. int/hiv/topics/comorbidities/about/en/

4. Nlooto M. Comorbidities of HIV infection and health care seeking behavior among HIV infected patients attending public sector healthcare facilities in KwaZulu-Natal: A cross sectional study. PLoS One. 2017;12:e0170983.

5. Margolis AM, Heverling H, Pham PA, et al. A review of the toxicity of HIV medications. J Med Toxicol. 2014;10(1):26-39.

6. Ward T, Sugrue D, Hayward O, et al. Estimating HIV Management and Comorbidity Costs Among Aging HIV Patients in the United States: A Systematic Review. J Manag Care Spec Pharm. 2020 Feb;26(2):104-16.

7. Katlamaa C, Ghosn J, Murphy R. Individualized antiretroviral therapeutic approaches: less can be more. AIDS. 2017, 31:1065-71.

8. Cahn P, Madero JS, Arribas JR, et al. Dolutegravir plus lamivudine versus dolutegravir plus tenofovir disoproxil fumarate and emtricitabine in antiretroviral-naive adults with HIV-1 infection (GEMINI-1 and GEMINI-2): week 48 results from two multicentre, double-blind, randomised, non-inferiority, phase 3 trials. Lancet. 2019 Jan 12;393(10167):143-55.

9. Cahn P, Madero JS, Arribas JR, et al. Durable Efficacy of Dolutegravir Plus Lamivudine in Antiretroviral TreatmentNaive Adults With HIV-1 Infection: 96-Week Results From the GEMINI-1 and GEMINI-2 Randomized Clinical Trials. J Acquir Immune Defic Syndr. 2020 Mar 1;83(3):310-08.
10. van Wyk J, Ajana F, Bisshop F, et al. Efficacy and Safety of Switching to Dolutegravir/Lamivudine Fixed-Dose Two-Drug Regimen Versus Continuing a Tenofovir Alafenamide-Based Three- or Four-Drug Regimen for Maintenance of Virologic Suppression in Adults With HIV-1: Phase 3, Randomized, Non-inferiority TANGO Study. Clin Infect Dis. 2020 Jan 6. pii: ciz1243.

11. Lopes S, O'Day K, Meyer K, et al. Comedication prescription patterns and potential for drug-drug interactions with antiretroviral therapy in people living with human immunodeficiency virus type 1 infection in Germany. Pharmacoepidemiol Drug Saf. 2020 Mar;29(3):270-78.

12. EACS Guidelines version 10.0. Published November 2019. Accessed 06 April 2020. https://www.eacsociety.org/ guidelines/eacs-guidelines/eacs-guidelines.html

13. Anderson SJ, Hsu CY, Ou HT, et al. Pharmacoeconomic evaluation of JULUCA (DTG/RPV) for human immunodeficiency virus (HIV-1) infection treatment in virologically-supressed adults in Taiwan. Value in Health. 2021 May;34:216-23.

14. Mauskopf J. A methodological review of models used to estimate the cost effectiveness of antiretroviral regimens for the treatment of HIV infection. Pharmacoeconomics. 2013;31(11):1031-50.

15. ViiV Healthcare data on file. CostEffectiveness Model for Dolutegravir (ARAMIS-DTG) in Treatment-Experienced HIV Patients Report Version 3.0. 2013.

16. ICER's Reference Case for Economic Evaluations: Principles and Rationale. Accessed on June 6, 2020. ICER. https:// icer.org/wp-content/uploads/2020/10/ ICER_Reference_Case_013120.pdf

17. Radford M, Parks DC, Ferrante S, et al. Comparative efficacy and safety and dolutegravir and lamivudine in treatment naive HIV patients. AIDS. 2019 Sep 1;33(11):1739-49.
18. Nickel K, Halfpenny NJ, Snedecor SJ, et al. Comparative efficacy and safety of a combination therapy of dolutegravir and lamivudine vs three drug antiretroviral regimens in treatment-naive HIV-1 infected patients at 96 weeks: a systematic review and network metaanalysis. Presented at AIDS 2020 Virtual Conference. Accessed on September 19, 2020. https://cattendee.abstractsonline. com/meeting/9289/presentation/106

19. Antinori A, Lazzarin A, Uglietti A, et al. Efficacy and safety of boosted darunavir-based antiretroviral therapy in HIV-1-positive patients: results from a meta-analysis of clinical trials. Scientific Reports. 2018;8(1):5288.

20. Baril J-G, Angel JB, Gill MJ, et al. Dual therapy treatment strategies for the management of patients infected with HIV: a systematic review of current evidence in ARV-naive or ARV-experienced, virologically suppressed patients. PLoS One. 2016;11:e0148231.

21. Kanters S, Socias ME, Paton NI, et al. Comparative efficacy and safety of second-line antiretroviral therapy for treatment of HIV/AIDS: a systematic review and network meta-analysis. The Lancet HIV. 2017 Oct;4(10):e433-e441. doi: 10.1016/S2352-3018(17)30109-1.

22. Cooper DA, Steigbigel RT, Gatell JM, et al. Subgroup and resistance analyses of raltegravir for resistant HIV-1 infection. NEJM. 2008;359:355-65.

23. Steigbigel RT, Cooper DA, Kumar PN, et al. Raltegravir with optimized background therapy for resistant HIV-1 infection. NEJM. 2008;359:339-54.

24. Despiegel N, Anger D, Martin M, et al. Cost-Effectiveness of Dolutegravir in HIV-1 Treatment-Naive and TreatmentExperienced Patients in Canada. Infect Dis Ther. (2015) 4:337-53.

25. Piscaglia M, Gallazzi, I., Restelli, S, et al. Impact on bone mineral density after two years of switching to four of switching to four dolutegravir dolutegravir-based triple or dual regimens. HIV Drug Therapy. 2018:P162. Accessed 06 April 2020. http://hivglasgow. org/2018-posters 
26. National Vital Statistics Report. United States Life Tables, 2015. Published November 13, 2018. Accessed 06 April 2020. https://www.cdc.gov/nchs/data/ nvsr/nvsr67/nvsr67_07-508.pdf

27. Lewden C CG, Morlat P, Raffi F, et al. HIV-infected adults with a CD4 cell count greater than 500 cells $/ \mathrm{mm}^{3}$ on longterm combination antiretroviral therapy reach same mortality rates as the general population. J Acquir Immune Defic Syndr. 2007;46:72-77.

28. Rydzak CE, Cotich KL, Sax PE, et al. Assessing the performance of a computerbased policy model of HIV and AIDS. PLoS One. 2010;5:e12647

29. d'Arminio Monforte A, Sabin CA, et al. The changing incidence of AIDS events in patients receiving highly active antiretroviral therapy. Arch Intern Med. 2005 Feb 28;165(4):416-23.

30. Szende A. Self-Reported Population Health: An International Perspective based on EQ-5D. In: Szende A, Janssen B, Cabases J, editors. Dordrecht: Springer; 2014.

31. D’Agostino RB, Vasan RS, Pencina MJ, et al. General cardiovascular risk profile for use in primary care. Circulation. 2008;117:743-53.

32. Kanis J, Johnell O, Oden A, et al. Ten year probabilities of osteoporotic fractures according to BMD and diagnostic thresholds. Osteoporosis International. 2001;12(12):989-95.

33. Gianotti N1, Galli L, Poli A, et al. Estimated Glomerular Filtration Rate Trajectories in HIV-Infected Subjects Treated With Different Ritonavir-Boosted Protease Inhibitors and Tenofovir Disoproxil Fumarate or Abacavir. Medicine (Baltimore). May;95(22):e3780.

34. MDDB US Product Price Reporting System. Accessed April 6, 2020.

35. Nshimyumukiza L, Durand A, Gagnon M, et al. An economic evaluation: Simulation of the costeffectiveness and cost-utility of universal prevention strategies against osteoporosis-related fractures. J Bone Miner Res. 2013;28(2):383-94.
36. Simpson KN, Pei PP, Moller J, et al. Lopinavir/Ritonavir Versus Darunavir Plus Ritonavir for HIV Infection: A CostEffectiveness Analysis for the United States. PharmacoEconomics. 2013 31: 427-44 doi: 10.1007/s40273-013-0048-43

37. Farnham PG, Gopalappa C, Sansom SL, et al. Updates of lifetime costs of care and quality-of-life estimates for HIV-infected persons in the United States: late versus early diagnosis and entry into care. J Acquir Immune Defic Syndr. 2013 Oct 1; 64(2):183-89.

38. Consumer Price Index for All Urban Consumers: Medical Care in US City Average (CPIMEDSL). US Bureau of Labor Statistics. Accessed May 9, 2020. https:// fred.stlouisfed.org/series/CPIMEDSL

39. Brogan AJ, Talbird SE, Cohen C. Cost-effectiveness of nucleoside reverse transcriptase inhibitor pairs in efavirenzbased regimens for treatment-naive adults with HIV infection in the United States. Value in Health. 2011;14:657-64.

40. Brogan AJ, Smets E, Mauskopf JA, et al. Cost effectiveness of darunavir/ritonavir combination antiretroviral therapy for treatment-naive adults with HIV-1 infection in Canada. Pharmacoeconomics. 2014;32:903-17.

41. Peng S, Tafazzoli A, Dorman E, et al. Cost-effectiveness of DTG $+\mathrm{ABC} / 3 \mathrm{TC}$ versus EFV/TDF/FTC for first-line treatment of HIV-1 in the United States. J Med Econ. 2015;18:763-76

42. Walensky RP, Sax PE, Nakamura YM, et al. Economic savings versus health losses: the cost-effectiveness of generic antiretroviral therapy in the United States. Ann Intern Med. 2013;158:84-92.

43. Girouard MP, Sax PE, Parker RA, et al. The cost effectiveness and budget impact of 2-drug dolutegravir-lamivudine regimens for the treatment of HIV infection in the United States. Clin Infect Dis. 2016;62(6):784-91.

44. HIV in United States and dependent areas. Centers for Disease Control and Prevention. Accessed December 10, 2020. https://www.cdc.gov/hiv/statistics/overview/ataglance.html
45. Sax PE, Meyers JL, Mugavero M, et al. Adherence to Antiretroviral Treatment and Correlation with Risk of Hospitalization among Commercially Insured HIV Patients in the United States. PLoS One. 2012;7(2):e31591. Epub 2012 Feb 24. doi: 10.1371/journal.pone.0031591

46. Kangethe A, Polson M, Lord TC, et al. Real-World Health Plan Data Analysis: Key Trends in Medication Adherence and Overall Costs in Patients With HIV. J Manag Care Spec Pharm. 2019 Jan;25(1):88-93. doi: 10.18553/ jmcp.2019.25.1.088

47. Sutton SS, Magagnoli J, Hardin J. Impact of Pill Burden on Adherence, Risk of Hospitalization, and Viral Suppression in Patients With HIV Infection and AIDS Receiving Antiretroviral Therapy. Pharmacotherapy. 2016;36(4):385-401. Epub 2016 Apr 13. doi: 10.1002/phar.172 48. Markowitz N, Tidwell B, Lamerato L, et al. Impact of Tablet Burden and Antiretroviral Therapy (ART) Choice on Virologic Outcomes in Treatment Naive HIV+ Individuals Attending an Inner City Clinic. Open Forum Infectious Diseases, Fall 2017;4(suppl_1):S429.

49. Langness J, Cook PF, Gill J, et al. Comparison of Adherence Rates for Antiretroviral, Blood Pressure, or Mental Health Medications for HIV-positive Patients at an Academic Medical Center Outpatient Pharmacy. J Manag Care Spec Pharm. 2014 Aug;20(8):809-14. doi: 10.18553/jmcp.2014.20.8.809

50. Blanco JL, Montaner JSG, Marconie VC, et al. Lower Prevalence of Drug Resistance Mutations at First-Line Virological Failure to First-Line Therapy With Atripla vs Tenofovir + emtricitabine/ lamivudine $\mu+$ Efavirenz Administered on a Multiple Tablet Therapy. AIDS. 2014;28:2531-9. doi: 10.1097/ QAD.0000000000000424

51. Priest J, Bhak R, DerSarkissian M, et al. Decreasing Adherence to Antiretroviral Therapy over 4 Years of Follow-up in a Commercially-Insured Population of Patients with HIV. Open Forum Infect Dis. October 2019;6(Suppl 2):S872. 
52. Sutton AJ, Welton NJ, Cooper N.

Evidence synthesis for decision making in healthcare: John Wiley \& Sons; 2012.

53. Smit M, Cassidy R, Cozzi-Lepri A, et al. Projections of non-communicable disease and health care costs among HIVpositive persons in Italy and the U.S.A.: A modelling study. PLoS One. 2017 Oct 23;12(10):e0186638.
54. Golestaneh L, Alvarez PJ, Reaven NL, et al. All-cause costs increase exponentially with increased chronic kidney disease stage. Am J Manag Care. 2017;23(10 Suppl):S163-s172.
55. Morris BL, Scott CA, Wilkin TJ, et al. Cost-effectiveness of adding an agent that improves immune responses to initial antiretroviral therapy (ART) in HIV-infected patients: guidance for drug development. HIV Clin Trials. 2012;13(1):1-10. 